\title{
FAIR status and the PANDA experiment
}

\section{A. Belias on behalf of the PANDA collaboration at FAIR}

GSI Helmholtzzentrum für Schwerionenforschung, D-64291 Darmstadt, Germany

E-mail: a.belias@gsi.de

ABSTRACT: The international accelerator Facility for Antiproton and Ion Research in Europe (FAIR) is the next generation accelerator complex for fundamental and applied research with antiproton and ion beams. FAIR will provide worldwide unique facilities enabling a wide spectrum of unprecedented forefront research in hadron and nuclear physics, in atomic physics and nuclear astrophysics as well as in applied sciences like materials research, plasma physics and radiation biophysics. Key features of FAIR are intense beams of antiprotons and ions up to the heaviest and even exotic nuclei covering an energy range from rest up to $30 \mathrm{GeV} / \mathrm{u}$. We present a brief overview on the current construction status of the FAIR accelerator facilities and the associated research pillars with emphasis on PANDA. PANDA (antiProton Annihilation in Darmstadt), is the central experiment to fully exploit the physics research potential of the High Energy Storage Ring (HESR) with intense, phase-space cooled, antiprotons up to $15 \mathrm{GeV} / \mathrm{c}$ impinging on a variety of fixed targets. The PANDA detector features two spectrometers, the Target Spectrometer with a SC solenoid magnet of $2 \mathrm{~T}$ and the Forward Spectrometer with a $2 \mathrm{Tm}$ dipole magnet. In both spectrometers the PANDA collaboration employs a multitude of modern detector technologies to provide tracking, particle identification, calorimetry and muon identification, arranged hermetically close to $4 \pi$ around the interaction region with additional detectors for coverage of the forward boosted particles. Focusing on the various PANDA detector systems we present an overview of recent developments, the detector construction progress and conclude with an outline for a phased deployment of PANDA at FAIR.

Keywords: Detector design and construction technologies and materials; Instrumentation for particle accelerators and storage rings - high energy (linear accelerators, synchrotrons); Calorimeters; Spectrometers 


\section{Contents}

1 Introduction 1

2 FAIR complex $\quad 2$

2.1 FAIR civil construction 3

2.2 FAIR accelerators procurement 4

3 FAIR science pillars $\quad 6$

3.1 APPA 6

3.2 CBM 6

3.3 NUSTAR

3.4 PANDA

4 The PANDA experiment at FAIR $\quad 8$

4.1 Antiprotons at FAIR 8

4.2 The PANDA detector 9

4.2.1 PANDA targets and magnets 9

$\begin{array}{lll}4.2 .2 & \text { PANDA tracking } & 10\end{array}$

$\begin{array}{lll}\text { 4.2.3 PANDA PID } & 11\end{array}$

$\begin{array}{lll}\text { 4.2.4 PANDA calorimetry } & 12\end{array}$

4.2.5 PANDA DAQ and controls $\quad 12$

$\begin{array}{lll}\text { 4.2.6 PANDA planning } & 13\end{array}$

$\begin{array}{lll}\text { 4.2.7 PANDA phase } 0 \text { program } & 13\end{array}$

5 Outlook 13

\section{Introduction}

The international Facility for Antiproton and Ion Research in Europe (FAIR), is currently under construction at Darmstadt next to the GSI facilitiy, the Helmholtz Centre for Heavy Ion Research, in Germany. FAIR is a multipurpose accelerator facility that will provide beams, of protons up to uranium ions, over a wide range of intensities and energies, to produce high intensities of high quality secondary beams of antiprotons and rare isotopes. FAIR is pushing the intensity frontier, complementary to the energy frontier for hadron beams at CERN. The FAIR consortium consists of nine international shareholders, from the following countries: Finland, France, Germany, India, Poland, Romania, Russia, Slovenia and Sweden, and includes one associated partner, the United Kingdom, and one aspirant partner, the Czech Republic. World-wide about 3000 scientists including about 500 $\mathrm{PhD}$ students will carry out experiments at FAIR to understand the fundamental structure of matter, to explore its exotic forms, and to understand how the universe evolved from its primordial form. 
The broad scientific program of FAIR comprises hadron physics, nuclear structure and astrophysics, atomic physics, plasma physics, materials research, and radiation biophysics and its applications in cancer therapy and space research. The science program is structured into four research pillars and organised in four large collaborations with several hundred members each: APPA, serving communities in Atomic, Plasma Physics and Applications; CBM, the Compressed Baryonic Matter experiment; NUSTAR, the NUclear STructure, Astrophysics and Reactions program; and PANDA (antiProton ANihilation in DArmstadt), the key experiment of the hadron physics program using antiproton beams at FAIR.

In this paper we present FAIR in section 2 with brief accounts on the current status of civil construction in section 2.1, and the accelerators in section 2.2, followed by an overview for each of the FAIR science pillars in section 3. Further emphasis is given to the PANDA experiment in section 4 with an overview on the status of the PANDA detector systems. We conclude with the current deployment plans of PANDA and the outlook to FAIR.

\section{FAIR complex}

The future FAIR accelerators [1], the existing GSI facility [2] along with several of the planned experiment locations are shown in figure 1.

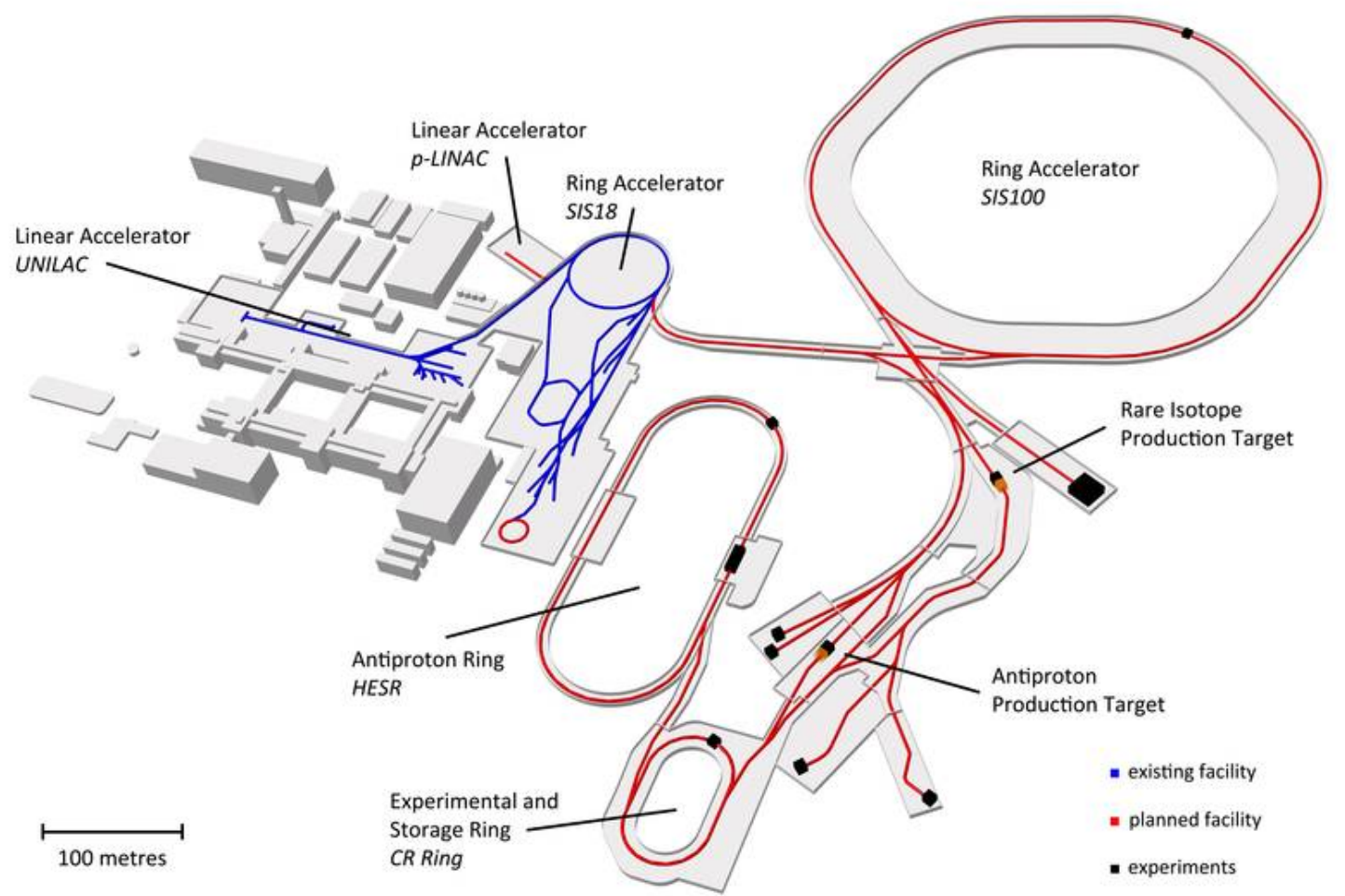

Figure 1. The existing GSI accelerators (blue) and the FAIR complex (red) with experiment locations (black). Graphic: GSI/FAIR.

The FAIR accelerator complex comprises the heavy-ion superconducting synchrotron SIS100 [3]; the Collector Ring (CR) [4]; High-Energy Storage Ring (HESR) [5]. The existing 
GSI accelerators, the heavy ion linac UNILAC [6] and the heavy ion synchrotron SIS18 [7], after a range of upgrades, will serve as injectors and boosters for SIS100. The fast-ramping SIS100 synchrotron is the centrepiece of FAIR and will provide intense primary beams for two production targets to generate new exotic nuclei and antiproton beams [8]. Secondary beams of rare isotopes and exotic nuclei, will be separated by the Superconducting FRagment Separator (Super-FRS) [9] to serve branches for different experimental areas and also for storage rings, while the antiproton separator selects and steers antiprotons to the collector ring (CR), with the main tasks their collection and cooling to inject the high-energy storage ring (HESR) for accumulation and cooling to provide high-intensity beams for in-ring experiments. Concerning the antiproton beams production, a new linear accelerator, the proton linac $[10,11]$ (pLinac) will be installed for high-intensity injection into the SIS18/SIS100 synchrotron chain. Both, the proton linac and also the CRYRING [12] which is already installed at GSI, while located on the existing GSI site, are part of the FAIR complex. Compared to GSI, the new accelerators at FAIR will deliver intense and energetic beams of particles, with up to $100-1000 \times$ higher intensities and $10 \times$ higher energies. The SIS100, with a circumference of $1.1 \mathrm{~km}$ and with a maximum magnetic bending power of $100 \mathrm{Tm}$, is designed to accelerate ion beams with maximum intensities ranging from $4 \times 10^{13}$ protons at $29 \mathrm{GeV}$ to $5 \times 10^{11}$ uranium ions at $2.7 \mathrm{GeV} / \mathrm{u}$. After production and separation, the resulting beams of secondary antiprotons or ion beams will drive several experimental scenarios either steered toward various fixed-target experiments or injected into the system of cooler and storage rings (CR and HESR) for novel in-ring experiments. In addition to the production of secondary beams, the primary beams will also serve fixed target interactions experiments. To drive such multitude of various experimental scenarios a complex of beam line sections the High Energy Beam Transport HEBT system [13] is planned with a total length of about $1.5 \mathrm{~km}$. The HEBT system is designed such that to enable switching between sections to serve experiments in a time sharing mode, allowing parallel operations.

\subsection{FAIR civil construction}

Tremendous progress has been made in the civil construction, considering the project was completely reorganised and consolidated in 2015, since the groundbreaking event on 4 July 2017. Aspects of construction progress are shown in figure 2.
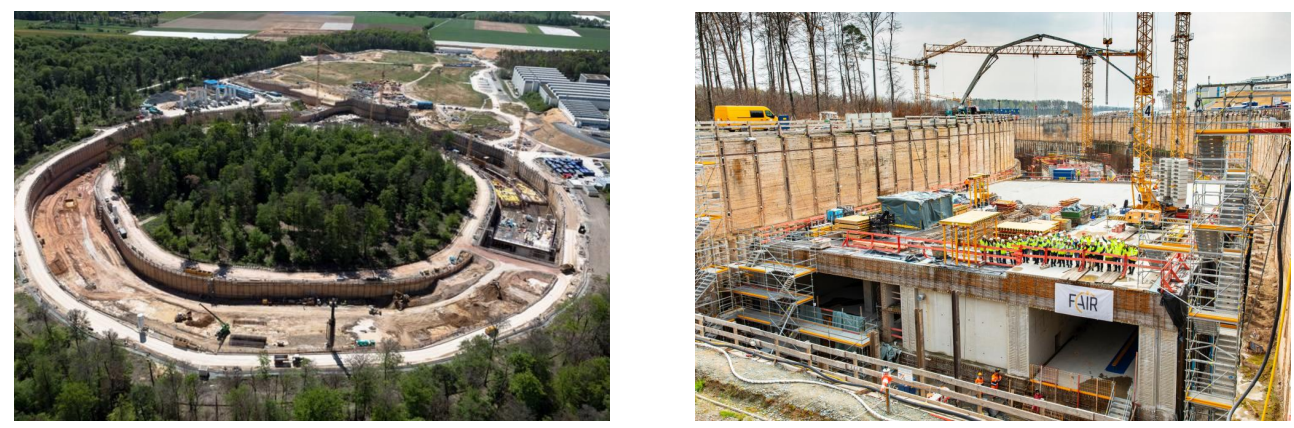

Figure 2. (Left) Birds-eye-view of FAIR civil construction; the SIS100 site. (Right) A section of the SIS100 tunnel construction. Images: GSI Helmholtzzentrum für Schwerionenforschung GmbH.

The civil construction plans include all buildings for accelerator and experimental structures, laboratories, and other operational and supply structures to be built on an area of approximately 
$150,000 \mathrm{~m}^{2}$ adjacent to the GSI site. Given the size and complexity of the FAIR project an integrated construction workflow planning is in place to closely coordinate civil construction works such as excavations, retaining walls, cement pouring, transport logistics, allowing for multishift operations at several areas in parallel. Outstanding progress has been made in the construction of the 1100 $\mathrm{m}$ double long tunnel, at a depth of $17 \mathrm{~m}$ below ground level, that will house the main accelerator SIS100 and a supply tunnel, both being built by the cut-and-cover construction method in about 200 $\mathrm{m}$ long sections enabling concurrent construction at different sections of the ring. Further notable progress made in civil construction works concern the GSI interface building to the FAIR tunnel including the new escape tunnel and the construction, carrying the additional soil shielding on top. Noteworthy as well is the progress on the excavations for the transfer building and of the area for the CBM experiment. While the current civil construction works are ongoing, the tenders to award the construction work to the remaining FAIR buildings are in preparation. For a most up-to-date information, including videos of the FAIR construction site, the reader is invited to visit the FAIR website, at [14].

\subsection{FAIR accelerators procurement}

Construction and procurement of the FAIR accelerator components and construction of associated system instrumentation is progressing continuously. Here we present just a snap-shot of procurement and tests in progress at a time prior to preparing the current contribution, based on recent presentations and publications [15]. A few photos of progress in the construction and the procurement of accelerator components are shown in figure 3 and tests in figure 4 .
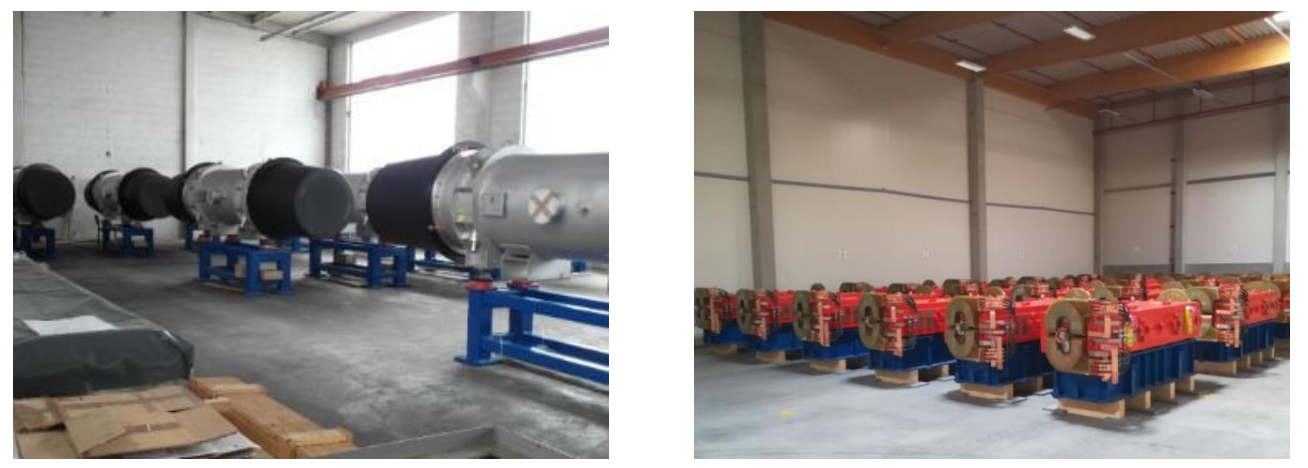

Figure 3. Accelerator components ready for installation. (Left) Superconducting dipole magnets in storage for the SIS100. (Right) Dipole magnets in storage for the High Energy Storage Ring (HESR). Images: GSI Helmholtzzentrum für Schwerionenforschung GmbH.

SIS100. Production of the superconducting dipole magnets for the SIS 100 is well in progress with about 60 (out of 110) modules manufactured at BNG and 55 were shipped to GSI and tested. Also, the SIS100 quadrupole units shipped from JINR to BNG for integration into FoS modules. The FoS vacuum chambers for the quadrupole doublet modules of the SIS100 also arrived from China and will be installed by the integrator in the quadrupole units. 
HEBT. The complete first batch of all 51 vacuum chambers for the HEBT has been delivered (BINP, Russia). Also, the series production of RF debunchers is progressing. The first of six series Power Converter from India, (ECIL, India) have arrived at GSI.

Super-FRS. First-of-Series of the Super-FRS short SC Multiplet arrived in 2019 at the CERN test facility for execution of the Site Acceptance Test (SAT). Successfully First-of-Series Factory Acceptance Tests (FAT) for the Super-FRS short SC Multiplet took place in Italy at January 2019.

pLinac. Copper plating and first tests of the RFQ accelerator cavity for the pLinac have been completed and match specifications.

HESR. Concerning the HESR, most of the components are manufactured, including all dipole and all quadrupole magnets, and all main quadrupole power converters. The vacuum chambers are presently being integrated into the magnets and more than $66 \%$ of the dipoles are delivered to FAIR, see figure 3. for storage until their installation. At COSY, various accelerator components are operated in beam tests including a prototype barrier bucket cavity and a FoS kicker and pick-up tanks for stochastic cooling.
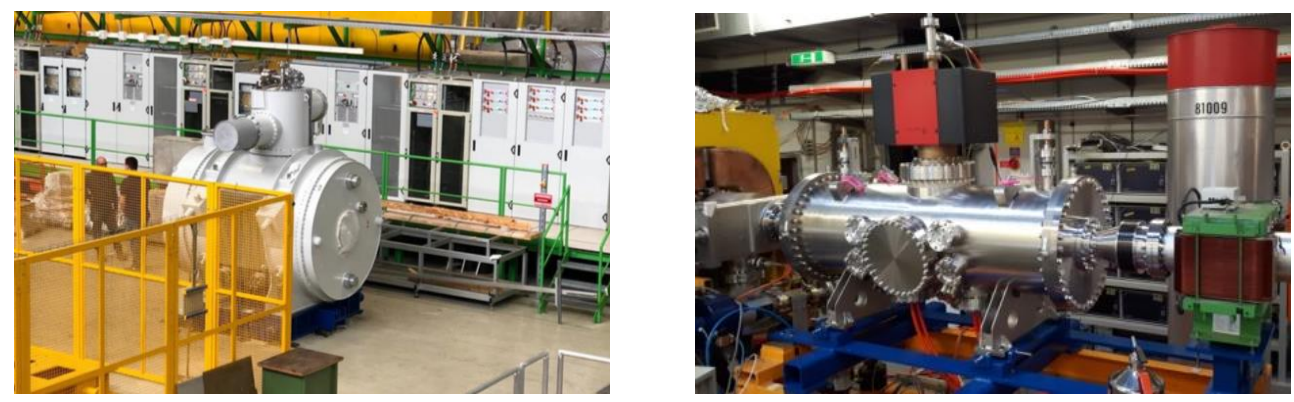

Figure 4. Accelerator components tests. (Left) FoS of the Super-FRS short SC Multiplet at CERN test facility. Image: GSI Helmholtzzentrum für Schwerionenforschung GmbH. (Right) First HESR Stochastic cooling pick-up and kicker in operation at COSY. Image: Forschungszentrum Jülich.

Distributed testing infrastructure facilities have been established to assess the different types of superconducting magnets for FAIR in addition to GSI, at JINR/Dubna, at CERN and at INFN/ Salerno. Since the FAIR accelerators are served by the existing accelerators of GSI, the heavy ion linac UNILAC and the heavy ion synchrotron SIS18 are being upgraded [16], for operation as injectors and boosters, concurrently to the on-going FAIR construction. At the time of writing, the reference project schedule forecasts the first beams available in 2025, after completing the commissioning plans of the various beam line sections. The current phase throughout FAIR construction until the commissioning of the new accelerators with beam, is called FAIR phase 0, during which the existing GSI facilities will continue to provide beam times to scientific requests [17]. Already the upgrades of the GSI accelerators allow for extensive testing of FAIR components and also provide beam times for tests of novel FAIR instrumentation and thereby enabling an attractive intermediate research program in FAIR phase 0. For example, the upgrade to higher intensities from the SIS18 benefits the hadronic-matter experimental program of HADES (High Acceptance Di-Electron Spectrometer) [18] which will already employ instrumentation of FAIR experiments, CBM and PANDA, in 2020 [19-21]. 


\section{FAIR science pillars}

The science program at FAIR is structured in four pillars as follows (in alphabetical order): (a) APPA: serving communities in atomic, plasma physics and applications; (b) CBM: research of nuclear- and quark-matter; (c) NUSTAR: nuclear structure and nuclear astrophysics (d) and PANDA research of hadron structure and dynamics. Research programs in each pillar are organised in the respective collaborations [22] with several hundred members each. A recent account on the FAIR science pillars in detail can be found in [23], while here we provide a brief overview of the research objectives.

\subsection{APPA}

With about 800 members, Atomic and Plasma Physics, and Applied sciences, APPA [24] is an umbrella for several subcollaborations covering a wide field of science with basic research in atomic physics, plasma physics as well as in applied sciences including material, biological and medical applications and space research. In the dedicated APPA experimental area, the APPA cave with several experimental stations of several target stations, with flexibility in detector settings and beam shaping, will benefit from the high-intensity ion and proton beams of SIS100. Additionally, experimental stations at the storage rings, the CRYRING and HESR [25], as well at the trapping facility HITRAP, will allow the APPA community SPARC (Stored Particles Atomic Research Collaboration) [26, 27] to address further physics challenges. The atomic physics program includes high-precision tests of bound-state QED in the non-perturbative regime and the precise determination of fundamental constants such as the fine-structure constant, which involves very precise measurements of ions confined in a trap. Regarding plasma physics studies of dense plasmas are planned to test models of planetary and stellar structure. The APPA program also involves BIOMAT (Biology and Material Science) [28], comprising research in biophysics and material science. Such as to assess the risk of space missions for astronauts and electronic equipment by dedicated irradiation experiments with FAIR beams simulating the high-energy component of galactic cosmic radiation and the studies of materials under the simultaneous exposure of irradiation and pressure.

\subsection{CBM}

The Compressed Baryonic Matter experiment (CBM) [29] will investigate highly compressed nuclear matter in high-energy nucleus-nucleus collisions which will recreate the matter densities found in supernova explosions, the cores of neutron stars and neutron-star mergers. At the energies of the FAIR beams CBM will systematically explore QCD matter at large baryon densities with high accuracy and rare probes. The QCD phase diagram at large baryon densities and various temperatures will be studied which is expected to exhibit a rich structure such as a critical point, a phase transition between hadronic and partonic matter, or new phases such as quarkyonic matter. A comprehensive review of the CBM physics research program can be fund in [30]. With more than 500 participants, the $10 \mathrm{~m}$ long fixed-target experiment CBM comprises a large-aperture superconducting dipole magnet with several subsequent detector systems providing tracking and particle identification. The CBM experiment is designed to run at beam intensities as high as $10^{9}$ ions/s and highest interaction rates in the field. of up to $10 \mathrm{MHz}$, which is 3-4 orders of magnitude higher than the rates reached in other high-energy heavy-ion experiments, using very fast and 
radiation-hard detectors. Recent developments of the Silicon Tracking System and the Projectile Spectator Detector have been presented at this workshop [31, 32].

\subsection{NUSTAR}

The NUclear STructure, Astrophysics and Reactions (NUSTAR) science program [33, 34] will explore the structure and reaction properties of very rare radioactive ions produced for the first time by FAIR. A major goal of NUSTAR is to improve our knowledge of the synthesis and abundance of chemical elements, and it will probe the limits of nuclear stability (lifetimes, decays), with studies of ground state properties (masses, radii), the structure of excited states (shell structure, shapes), as well as unbound and other exotic systems (halo, skin) and the nuclear equation of state. FAIR offers unique beams for such studies, by artificially producing nuclei that occur in nucleosynthesis sites in the universe enabling studies of the radioactive intermediate products in the different processes involved in the formation of isotopes directly in the laboratory. The NUSTAR collaboration with more than 800 participants is structured in several subcollaborations with different experimental setups across FAIR. The Super-FRS will make very efficient use of the highly intense beams at high energies to separate beams of the heaviest and most neutron-rich nuclei to serve various experiment setups whereas the network of storage rings at FAIR will allow further, additional, mass and lifetime measurements. With many detector set-ups of NUSTAR well advanced or already complete, the collaboration plans installations in various new experimental halls starting from 2023.

\subsection{PANDA}

The antiProton ANihilation in DArmstadt (PANDA) [35, 36] is a unique experiment at FAIR dedicated to fully exploit the new dimension of beams which will be available at FAIR, the highquality antiproton beams at the high-energy storage ring (HESR). The PANDA physics program encompasses a wide range of fundamental research of hadron structure and dynamics and nuclear physics including searches and studies of exotic hadrons such as tetra- and pentaquarks, hybrids and glueballs, studies of quark dynamics and hadron dynamics such as hadrons inside nuclear media, and also proton form-factor measurements, deep virtual Compton scattering, as well as various exciting studies of strange quarks in hyperons. With the brilliant antiproton beams available at the HESR the following PANDA physics pillars will be covered extensively:

- Nucleon Structure

- Physics with Strangeness

- Charm Physics and Exotics

- Hadrons in Nuclei

Comprehensive overviews of the physics objectives with details of the PANDA physics program can be found in [37-41]. The PANDA collaboration consists of 500 scientists in 65 institutes from 18 countries, with a vibrant and exciting experiment program. 


\section{The PANDA experiment at FAIR}

The PANDA experiment at FAIR [42] features a modern multipurpose detector with excellent tracking, calorimetry and particle-identification capabilities, to exploit fully the high-quality antiproton beams at FAIR provided by the High Energy Storage Ring (HESR) at an unprecedented annihilation rate with sophisticated physics event selection.

\subsection{Antiprotons at FAIR}

The antiproton production chain at FAIR starts with the pLinac providing protons of $70 \mathrm{MeV}$ which are accelerated further in the SIS 18 to $4 \mathrm{GeV}$ and injected into SIS100. Every 10 seconds $2.5 \times 10^{13}$ protons will be accelerated in the SIS100 to $29 \mathrm{GeV}$ and compressed into a bunch of 50 ns. Antiprotons with energies around $3 \mathrm{GeV}$ will be produced through inelastic collisions of these high energy protons with nucleons of a metal target at rest. A pulsed magnetic horn will focus the antiprotons down the pbar separator which will accept only the antiprotons for transfer to the Collector Ring (CR). Fast cooling in the CR allows the efficient collection of antiproton bunches which are injected for accumulation and stochastic cooling and post-acceleration in the HESR. The HESR (see figure 5) is designed as a racetrack-shaped storage ring with a circumference of 575 $\mathrm{m}$ and magnetic bending power of $50 \mathrm{Tm}$. It is based on a lattice of normal-conducting magnets designed to deliver antiproton beams in the momentum range from 1.5 to $15 \mathrm{GeV} / \mathrm{c}$.

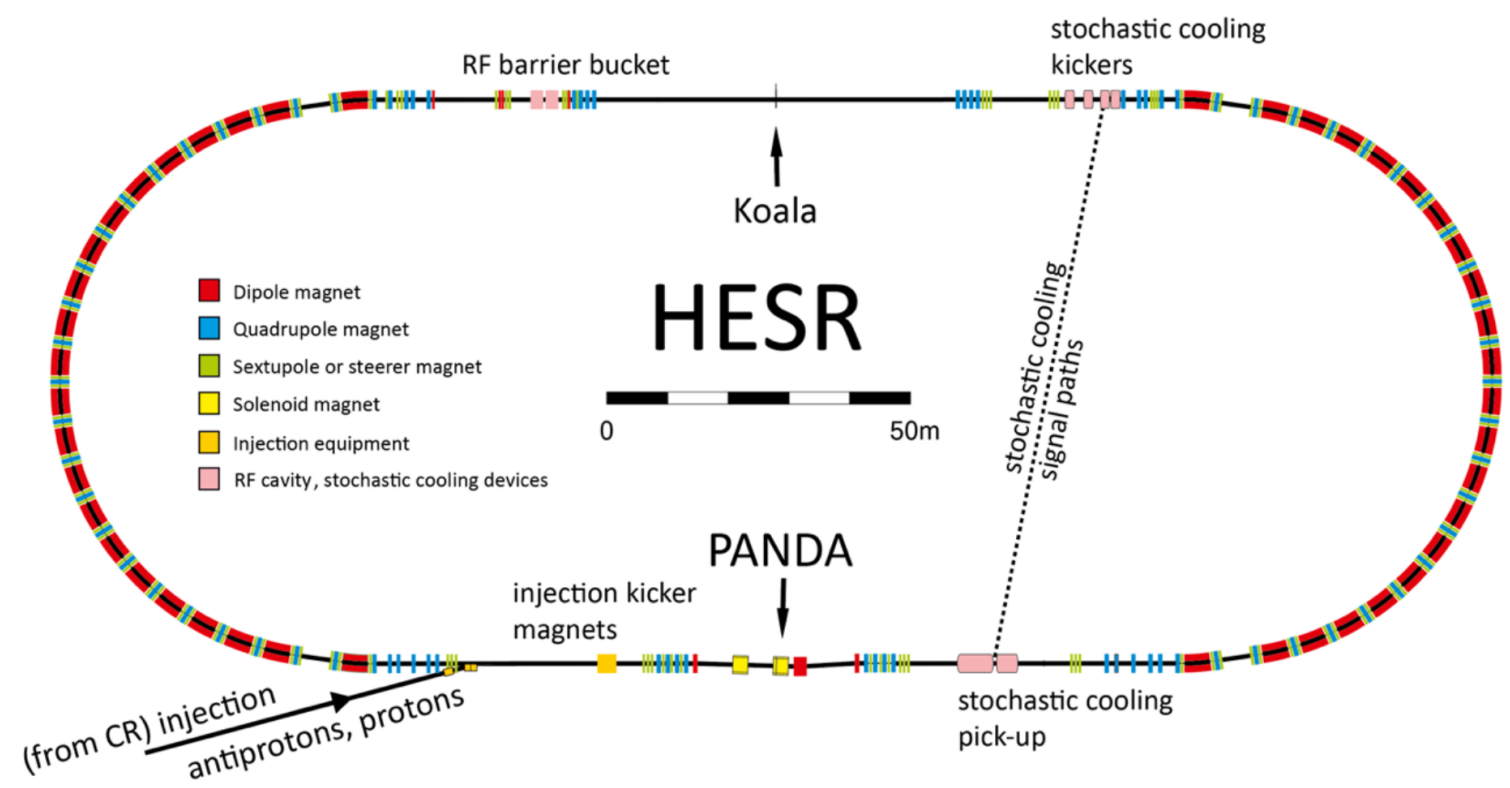

Figure 5. High Energy Storage Ring (HESR). Graphic: Forschungszentrum Jülich.

An important feature of this new facility is the high-bandwidth stochastic cooling [43] capable to provide phase-space cooled antiproton beams with which the PANDA internal targets (proton or nuclear targets) opens outstanding capabilities for high precision experiments in QCD [44]. Two operation modes are foreseen: a) the high luminosity mode with peak luminosities of up to $2 \times 10^{32} \mathrm{~cm}^{-2} \mathrm{~s}^{-1}$ and b) high resolution mode with a relative momentum spread of the order of a few $\times 10^{-5}$. Annihilations of antiprotons-protons have enormous advantages compared to proton-proton 
collisions, such as small momentum transfer at maximum released energy with well-defined initial states. Furthermore, the stochastic cooling available at HESR over the full momentum range, allows for production experiments with high-precision beam energy (about $50 \mathrm{keV}$ ) scans capable to reveal and study unknown features of underlying resonance structures with the PANDA detector [38].

\subsection{The PANDA detector}

PANDA is a fixed-target experiment which will be located in one of the straight sections at HESR inside the PANDA experiment building. The PANDA Detector (see figure 6) consists of two spectrometers, the Target Spectrometer (TS) with installation of an internal target within a superconducting solenoid magnet and the Forward Spectrometer (FS) with a dipole magnet.

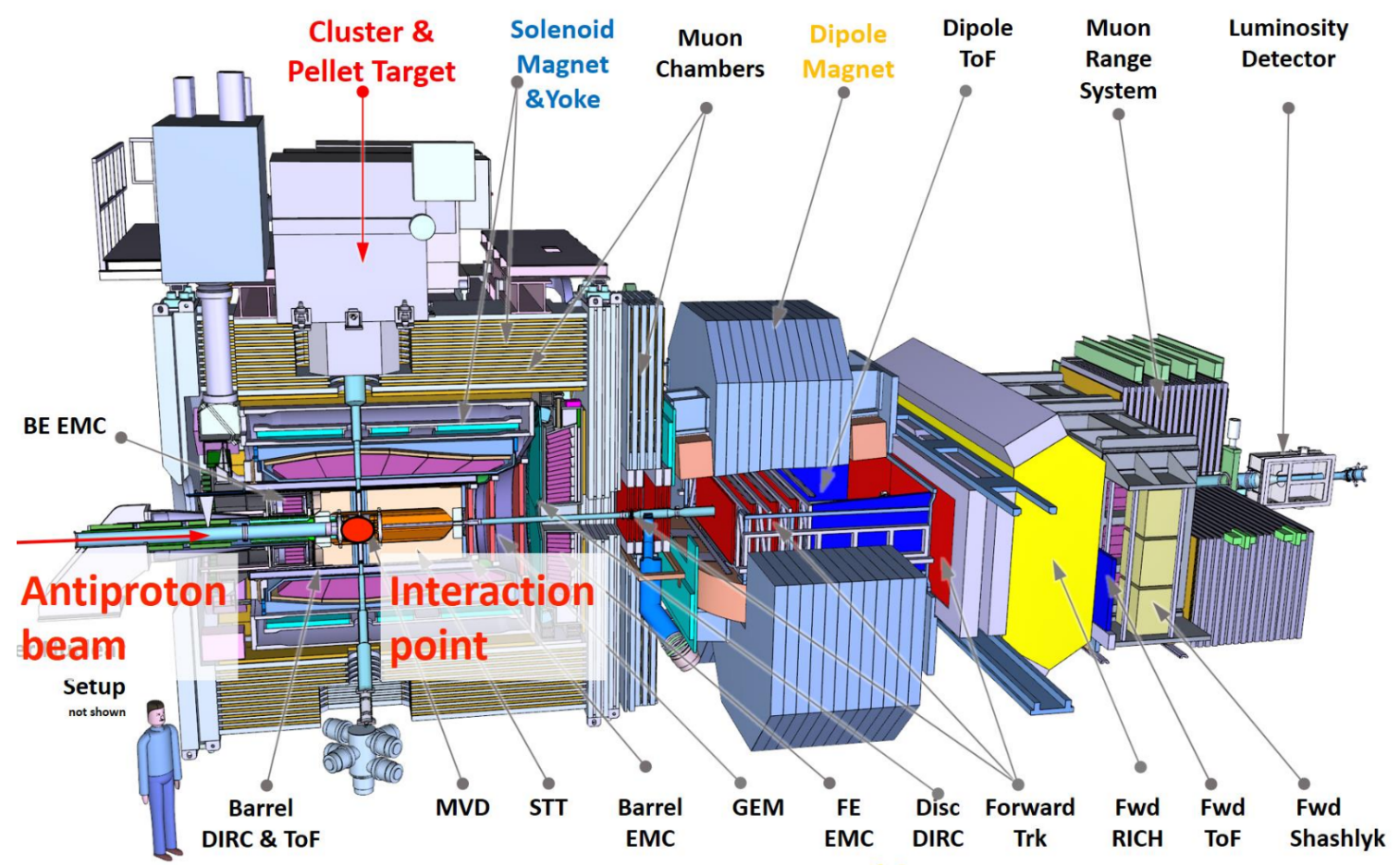

Figure 6. PANDA detector.

Both the TS and the FS are placed on movable support platforms, to move into the HESR beam, and out into the maintenance area of the PANDA hall. Versatile detector instrumentation in both spectrometers feature precise trajectory reconstruction, tracking, energy and momentum measurements, calorimetry and very efficient charged particle identification, PID. The detectors in the TS enclose the interaction point in a typical onion-like structure, similar to detector designs in collider experiments, providing almost $4 \pi$ coverage. While the detectors in the FS are arranged along the beam line to cover polar angles up to $\pm 10^{\circ}$ horizontally and $\pm 5^{\circ}$ vertically.

\subsubsection{PANDA targets and magnets}

A cluster jet or pellet target system will be used to provide either a cluster beam of a target gas or frozen hydrogen pellets at distance of about $2.1 \mathrm{~m}$ from the source to the interaction point. The 
Cluster Jet Target system [45] is very advanced with a prototype operating successfully at COSY (see figure 7) achieving world record densities of $4 \times 10^{15}$ atoms $\mathrm{cm}^{-2}$.

The technical design of the pellet target system is in progress to provide a constant stream of frozen hydrogen pellets smaller than $10 \mu \mathrm{m}$. Other target types and materials, such as thin foils or noble gases, are also planned in PANDA enabling a variety of novel antiproton-nucleus studies [46].
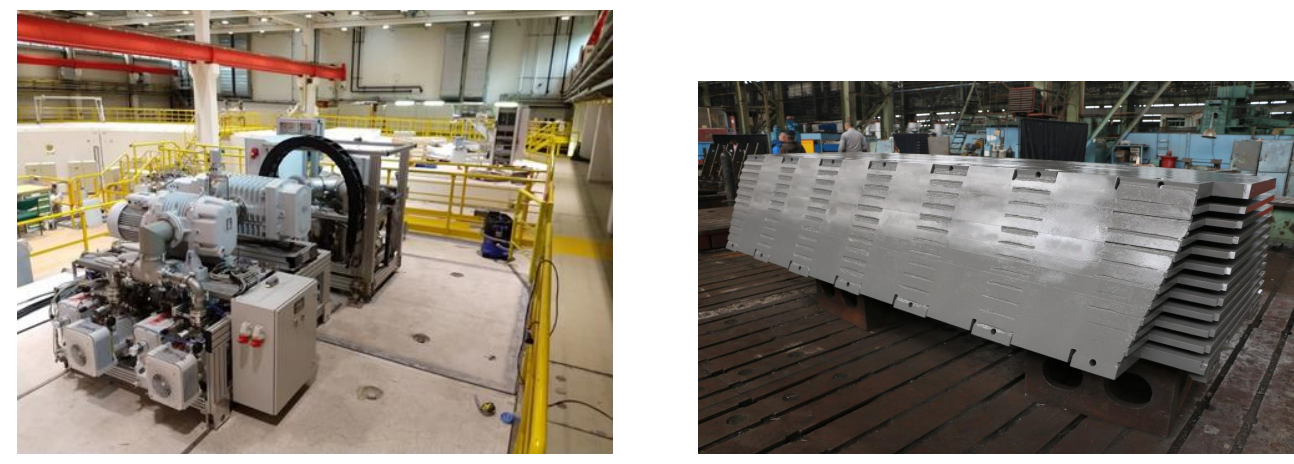

Figure 7. (Left) The PANDA Cluster Jet target prototype at COSY. (Right) Yoke octants produced for the superconducting solenoid in PANDA. Images: PANDA Annual Report 2019.

Both PANDA magnets are described in [47]. The superconducting solenoid magnet in the TS will provide a magnetic field up to $2 \mathrm{~T}$ (with a homogeneity of $\pm 2 \%$ ) in the beam direction. and the dipole magnet in the FS an integral field of $2 \mathrm{Tm}$. The solenoid with an inner bore of $1.9 \mathrm{~m}$ has a segmented coil allowing the target pipe to cross through. Production tests of a SC cable prototype by a joint venture of BINP and Russian manufacturer show good progress. The segmented yoke (see figure 7) and the laminated doors will be instrumented with chambers for muon identification. All octants of the yoke are produced and preparations for the first assembly are in progress [48]. Regarding the dipole magnet, which forms a part of the HESR beam line, the detailed design is being finalised and its production is due to start.

\subsubsection{PANDA tracking}

Closest to the interaction point a silicon based tracker, the Micro Vertex Detector (MVD) [49] is planned to provide vertex resolutions of about $50 \mu \mathrm{m}$ in transverse and $100 \mu \mathrm{m}$ along the beam direction. Pixel sensors, at the inner most layers and double-sided strips sensors will use ASICs developed in PANDA [50] with radiation hard front-end readout and power drivers developed at CERN [51, 52]. Surrounding the MVD, the Straw Tube Tracker (STT) and Gas Electron Multiplier (GEM) stations will be used for tracking charged particles with a transverse momentum resolution of about $1.2 \%$ in the magnetic field. The STT [53] is based on a self-supporting drift tube design, of aluminised Mylar tubes with a gold-plated tungsten wire, operated with $\mathrm{Ar} / \mathrm{CO}_{2}$ at overpressure, arranged in planar layers parallel to the beam.

At the FS, the Forward Tracking System [54] uses the same drift tube design, arranged in multiple straw tube layers perpendicular to the beam, such as shown in figure 8 , and located before, after, and within the dipole. Both tracking detectors, STT and FT will use the same ASICs developed in PANDA [55] with the front-end readout developments at GSI [56]. 

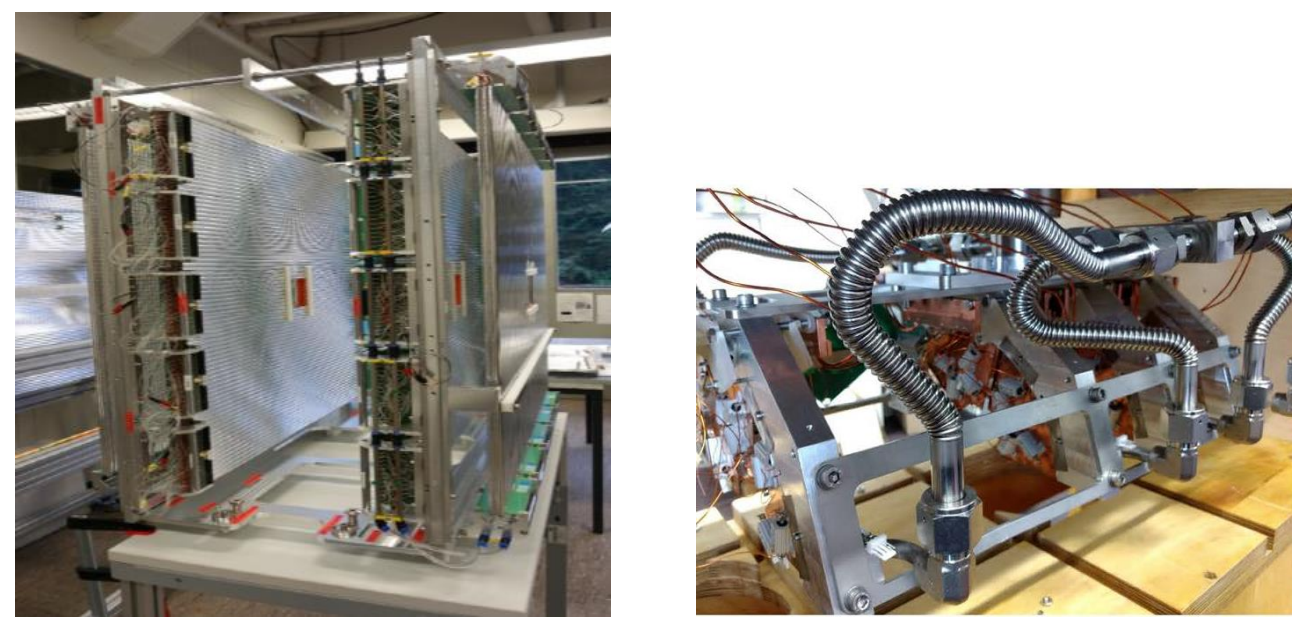

Figure 8. (Left) PANDA straw tube tracker stations. (Right) PANDA Luminosity detector prototype. Images: PANDA Annual Report 2019.

All the straws for the STT have been produced and the module production is in progress and also the in-kind contacts are signed for the production of the readout ASICs for both trackers.

At the far forward region, about $11 \mathrm{~m}$ from the IP, the Luminosity Detector (LMD) [57] uses elastic scattering of antiprotons on protons to determine the interaction rate by measuring antiprotons deflected at low angles. The LMD features retractable half-plane layers with HVMAPS in a secondary vacuum around the beam pipe, as shown in contributions to this workshop [58, 59]. Advanced prototypes, see figure 8 , fulfill or exceed the PANDA requirements and detector construction is due to start.

\subsubsection{PANDA PID}

Particle identification of pions, kaons and protons in the TS will utilize information from a Time-ofFlight system (ToF) [60], a Barrel DIRC (Detection of Internally Reflected Cherenkov light) [61], and an Endcap Disc DIRC detector [62], see figure 9.

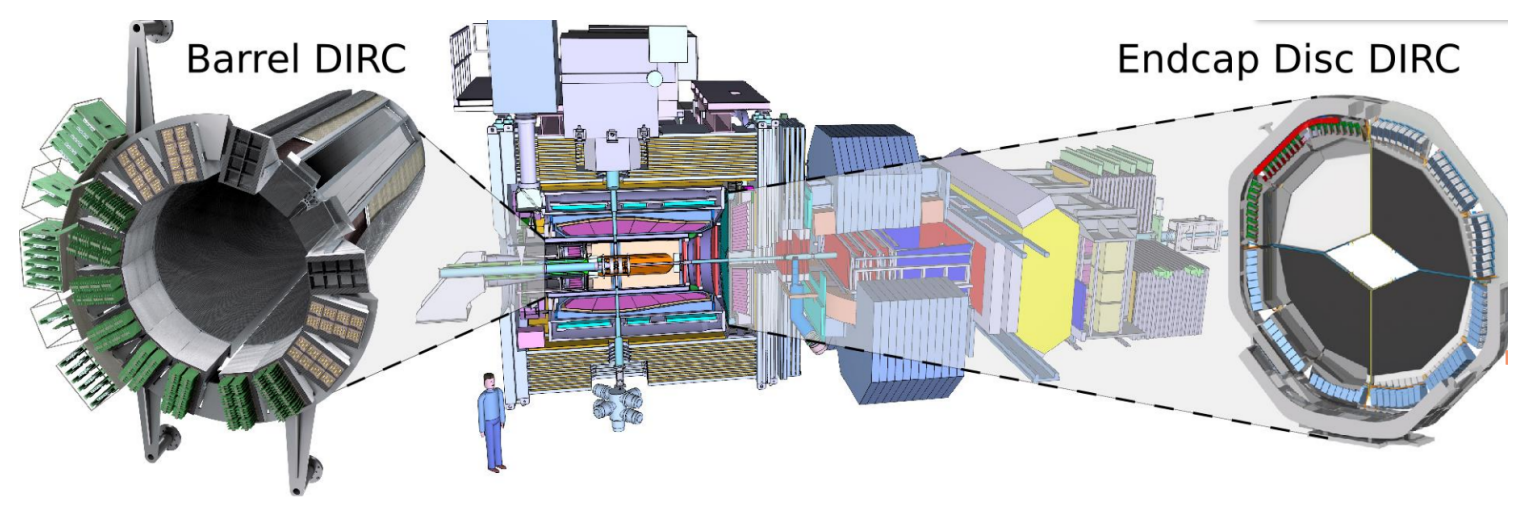

Figure 9. PANDA Barrel DIRC and Endcap Disc DIRC.

The Barrel DIRC is a bar-type DIRC with quartz prisms, arranged in sections of a barrel geometry while the Disc DIRC uses four large quartz plates perpendicular to the beam line. 
Advanced prototype studies have shown that both detectors meet the PID goal of 3 standard deviation pion/kaon separation up to 3.5 and $4 \mathrm{GeV} / \mathrm{c}$, respectively. Design and performance results of prototypes and the status of DIRC counters in PANDA can be found in contributions to this workshop [63, 64]. The ToF system in the TS will use scintillating tiles with Silicon Photomultiplier readout. A Forward Time-of-Flight wall [65] and an aerogel-based Ring Imaging Cherenkov Counter detector also presented in this workshop [66], will provide particle identification in the FS.

\subsubsection{PANDA calorimetry}

Photons and the energy of electrons will be reconstructed with the Electromagnetic Calorimeter (EMC) [67]. In the TS, the EMC consists of three distinct detectors, the Barrel EMC, the Forward Endcap EMC and the Backward Endcap EMC, with a total of about 16,000 $\mathrm{PbWO}_{4}$ crystals, to provide almost $4 \pi$ coverage, with an energy resolution of $1.5 \% / \sqrt{E}$, with $\mathrm{E}$ in units of $\mathrm{GeV}$. The high quality second-generation lead tungstate crystals used in PANDA will operate at -25 ${ }^{\circ} \mathrm{C}$ to further increase the light yield. The photosensors for the crystals are large area Avalanche PhotoDiodes (APDs), developed for PANDA, except for the high occupancy region in the Forward Endcap EMC centre where Vacuum Photo Tetrodes (VPTTs) are used. Production of all three EMC detectors is in progress, including a dedicated mass screening of crystals and APDs with pre-, and post-irradiation /annealing measurements. The Forward Endcap EMC construction (see figure 10) is well advanced, all crystals have been produced, with all VPTT modules produced, the APD modules production underway and with all front-end Sampling ADC boards ready, the pre-assembly of the full endcap is in preparation. The FoS of the 16 Barrel EMC slices has been produced, see figure 10, and construction of further slices is coordinated with the crystal production schedules. All pre-amp ASICs, designed at GSI [68] for the APD readout, are produced already. The Backward Endcap EMC construction has started with the procurement of components and APD sub-module production.

In the FS a Shashlyk-type Calorimeter [69] with an energy resolution of 3\%/ $\sqrt{E}$ is followed by the Muon Range System for muon detection [70]. The Shashlyk-type Calorimeter uses interleaved scintillator /absorber plates with WLS fibers coupled to PMTs and readout with FADC, as presented at this workshop [71]. Instrumentation for muon detection in both the TS and FS is based on drift tubes with wire and cathode strip readout by modern, newly developed FPGAs.

\subsubsection{PANDA DAQ and controls}

The Data AcQuisition (DAQ) is based on a triggerless readout scheme with flexible data selections to numerous physics channels. A new type of intelligent readout of the front-end electronics is being developed, where kinematical constraints are imposed online on reconstructed events. Recent TDC front-end developments at GSI [72] have also been presented at this workshop. A data reduction factor of up to about $10^{3}$ is expected to be achieved by employing this technique for the whole detector, resulting in a data rate of about $10^{4}$ events/s (or, equivalently, about $200 \mathrm{MB} / \mathrm{s}$ ) to be sent to storage for offline processing and analysis.

Controls, configuration, and monitoring for each detector system in PANDA, will be based on Experimental Physics and Industrial Control System (EPICS) [73]. This provides a layered approach with detector system specific drivers, connected to a PANDA-wide generic access layer 

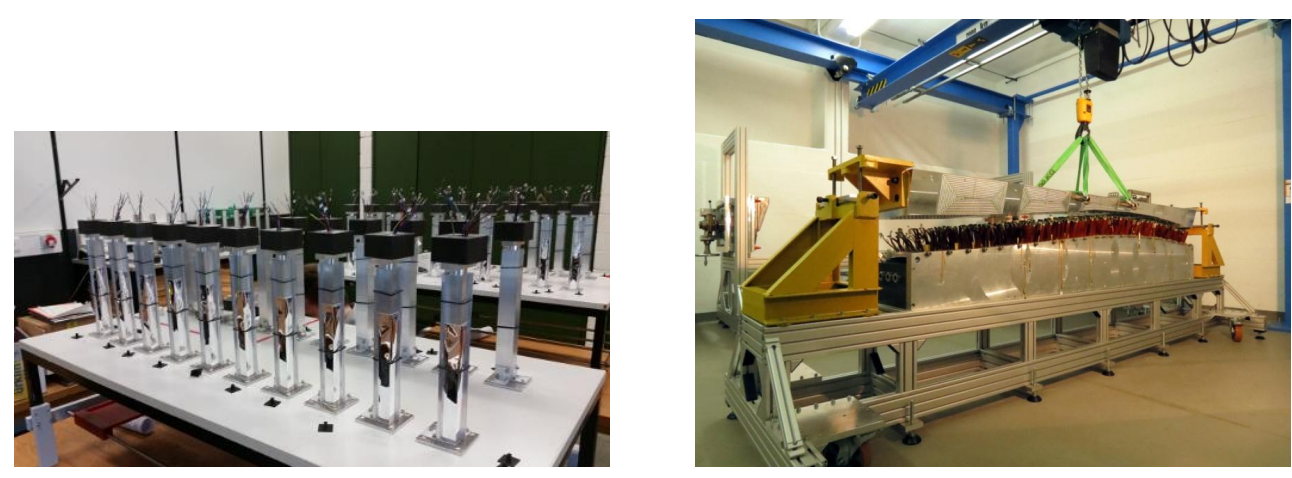

Figure 10. (Left) Forward Endcap EMC crystal setup. (Right) Barrel EMC FoS slice. Images: PANDA Annual Report 2019.

and a supervisory layer with interfaces to control and monitor GUIs for the shift crew on the status of the detector, DAQ and the HESR beam line.

\subsubsection{PANDA planning}

As with any large-scale project, a phased schedule has been implemented for FAIR and the PANDA experiment planing is fully embedded in the overall FAIR schedule. Most PANDA detector systems are currently in a procurement and construction phase, with completion envisaged by 2023 . The installation is planned as soon as the PANDA building is available, in two periods, (a) installation of large structures, the solenoid and dipole magnets and supports in 2022 and (b) installation of detector systems in 2023/2024. The first detector systems installed form the Day-1 configuration with the commissioning phase planned in 2024/2025 using proton beams to be followed by the phase 1 for physics with antiprotons in 2025/2026. At the same time construction and installation of further detector systems will complete the PANDA full setup in 2026, for physics with antiprotons in phase 2 and beyond. These plans were formulated prior to the worldwide coronavirus pandemic, which could lead to unexpected changes.

\subsubsection{PANDA phase 0 program}

While detector construction is in progress several PANDA detector groups are actively participating in the FAIR phase 0 program. Both the STT and FT groups have FAIR phase 0 activities, as mentioned in section. contributing tracker stations based on PANDA straw tube detector technologies to the HADES experiment at GSI. Further phase 0 activities of PANDA detector instrumentations include (a) the PANDA Luminosity Detector activities with the KOALA project at COSY [74], (b) the Backward Endcap EMC installation at MAMI [75]. and (c) the Barrel DIRC activities at the GlueX experiment at Jefferson Lab, also presented at this workshop [76].

\section{Outlook}

FAIR is rapidly developing, with major thrust on the construction of FAIR accelerators and experiments. Both civil construction and procurement of accelerator components proceed rapidly, and the experiments are getting ready. A staged approach to FAIR science and progressive commissioning 
of accelerators and detectors encompasses: the FAIR phase 0 , currently in progress, the FAIR phase 1 with start configurations of experiments in place and the accelerators progressively approaching design parameters in 2024/2025 and finally full FAIR operation in 2025/2026+. Antiproton beam facilities are firmly embedded in the FAIR planning and the PANDA experiment is fully integrated in the FAIR schedule. FAIR is a unique opportunity for world science, with a fascinating and broad science program, with world class and pioneering experiments.

\section{Acknowledgments}

The author would like to thank the INSTR20 conference committees for their kind invitation. Also, special thanks to all GSI/FAIR work package leaders, the department heads, Klaus Peters and Jochen Schwiening, the PANDA Technical Coordination team, Lars Schmitt, Udo Kurilla, Jost Lühning, Stefan Koch and Daniel Glaab and all colleagues and contributors for their relentless efforts towards realization of FAIR.

\section{References}

[1] FAIR GmbH, GSI, Green Paper, The Modularized Start Version: FAIR - Facility for Antiproton and Ion Research, GSI-2013-04816, Darmstadt, Germany (2009).

[2] GSI-FAIR (eds.), GSI-FAIR Scientific Report 2018, https://doi.org/10.15120/GSI-2019-00545.

[3] P. Spiller and G. Franchetti, The FAIR accelerator project at GSI, Nucl. Instrum. Meth. A 561 (2006) 305.

[4] A. Dolinskii, Collector Ring project at FAIR, (2015), https://doi.org/10.13140/RG.2.1.3096.3042.

[5] R. Maier et al., The high energy storage ring (HESR), proceedings of Particle Accelerator Conference PAC2011, New York, NY, U.S.A., 2011.

[6] W. Barth et al., Heavy Ion High Intensity Upgrade of the GSI UNILAC, AIP Conf. Proc. 773 (2005) 94.

[7] S. Sorge et al., Determination of the acceptance of Sis-18 using an RF Voltage, Conf. Proc. C 100523 (2010) THPEB003, IPAC-2010-THPEB003.

[8] K. Knie, B. Franzke, V. Gostishchev, M. Steck and P. Sievers, Concept for the Antiproton Production Target at FAIR, Conf. Proc. C 1205201 (2012) WEPPD030.

[9] H. Geissel et al., The Super-FRS project at GSI, Nucl. Instrum. Meth. B 204 (2003) 71.

[10] C. Kleffner et al., Status of the FAIR proton LINAC, Proc. LINAC2018 (2018) 787.

[11] L. Groening et al., The 70-MeV Proton Linac for the Facility for Antiproton and Ion Research FAIR, Proc. LINAC2006 (2006) 186.

[12] M. Lestinsky et al., Physics book: CRYRING@ESR, Eur. Phys. J. ST 225 (2016) 797.

[13] M. Schwickert et al., Beam Current Monitors for FAIR, Proc. IPAC2014 (2014) 3483.

[14] GSI-FAIR, Photos and videos of the FAIR construction site, https://www.gsi.de/en/researchaccelerators/fair/fair_civil_construction/photos_and_videos.htm (accessed 24 Feb 2020).

[15] P. Spiller et al., Status of the FAIR project, Proc. IPAC2018 (2018) 63. 
[16] P. Spiller et al., Status of the FAIR Synchrotron Projects SIS18 Upgrade and SIS100, Proc. IPAC2014 (2014) 1857.

[17] M. Bai et al., Challenges of FAIR Phase 0, Proc. IPAC2018 (2018) THYGBF3.

[18] HADES collaboration, The High-Acceptance Dielectron Spectrometer HADES, Eur. Phys. J. A 41 (2009) 243 [arXiv: 0902 . 3478].

[19] HADES, CBM and TRB collaborations, The HADES-RICH upgrade using Hamamatsu H12700 MAPMTs with DiRICH FEE + Readout, 2018 JINST 13 C03038.

[20] J. Smyrski et al., Pressure stabilized straw tube modules for the PANDA Forward Tracker, 2018 JINST 13 P06009.

[21] N. Rathod et al., Hyperon studies and development of forward tracker for hades detector, Acta Phys. Polon. B 51 (2020) 239.

[22] FAIR GmbH, Experiments - The Four Scientific Pillars of FAIR, https://fair-center.eu/for-users/experiments.html (accessed 24 Feb 2020).

[23] M. Durante et al., All the fun of the FAIR: fundamental physics at the facility for antiproton and ion research, Phys. Scripta 94 (2019) 033001 [arXiv: 1903.05693].

[24] APPA collaboration, APPA at FAIR: From fundamental to applied research, Nucl. Instrum. Meth. B 365 (2015) 680.

[25] R. Sáanchez et al., Towards experiments with highly charged ions at HESR, X Ray Spectrom. 49 (2020) 33.

[26] SPARC collaboration, SPARC: The Stored Particle Atomic Research Collaboration At FAIR, AIP Conf. Proc. 1336 (2011) 132.

[27] SPARC collaboration, Atomic physics experiments at the high energy storage ring, Phys. Scripta T 166 (2015) 014025.

[28] M. Durante, Y. Prezado and V. Patera, The Biophysics Collaboration for research at FAIR and other new accelerator facilities, Europhys. News 50 (2019) 27.

[29] CBM collaboration, Probing dense QCD matter in the laboratory - The CBM experiment at FAIR, Phys. Scripta 95 (2020) 074003 [arXiv: 2005. 03321].

[30] B. Friman et al. eds., The CBM physics Book, Lect. Notes Phys. 814, Springer (2011).

[31] CBM collaboration, Analysis of $2.7 \mathrm{GeV}$ proton-beam measurements with the STS detector for the CBM experiment, International Conference on Instrumentation for Colliding Beam Physics, Novosibirsk, Russia, 24-28 February, 2020.

[32] CBM collaboration The Readout system of the CBM Projectile Spectator Detector at FAIR, International Conference on Instrumentation for Colliding Beam Physics, Novosibirsk, Russia, 24-28 February, 2020.

[33] NUSTAR collaboration, The NUSTAR Project at GSI and FAIR, Exotic Nuclei (2015) 495 [DOI].

[34] NUSTAR collaboration, NUSTAR - The teenage years, Hyperfine Interact. 238 (2017) 35.

[35] K. Peters, L. Schmitt, T. Stockmanns and J. Messchendorp, PANDA: Strong Interaction Studies with Antiprotons, Nucl. Phys. News 27 (2017) 24.

[36] PANDA collaboration, Letter of intent for PANDA - Strong Interaction Studies with Antiprotons, Technical Report FAIR - ESAC (2004). 
[37] PANDA collaboration, The PANDA Experiment at FAIR - Subatomic Physics with Antiprotons, JPS Conf. Proc. 13 (2017) 010016 [arXiv: 1610.02804].

[38] M.F.M. Lutz et al., Resonances in QCD, Nucl. Phys. A 948 (2016) 93 [arXiv: 1511.09353$].$

[39] PANDA collaboration, Studies of Hadron Structure and Interactions with the $\bar{P} A N D A$ Experiment at FAIR, arXiv: 1512.03299.

[40] U. Wiedner, Future Prospects for Hadron Physics at PANDA, Prog. Part. Nucl. Phys. 66 (2011) 477 [arXiv: 1104.3961].

[41] PANDA collaboration, Physics Performance Report for PANDA: Strong Interaction Studies with Antiprotons, arXiv:0903.3905.

[42] PANDA collaboration, PANDA website, https://panda.gsi.de/ (accessed 24 Feb 2020).

[43] R. Stassen et al., Recent results of HESR original stochastic cooling tanks at COSY, J. Phys. Conf. Ser. 1067 (2018) 2013

[44] N_TOF collaboration, Cross section measurements of ${ }^{155,157} G d(n, \gamma)$ induced by thermal and epithermal neutrons, Eur. Phys. J. A 55 (2019) 9 [Erratum ibid. 55 (2019) 45] [arXiv: 1805 . 04149].

[45] PANDA collaboration, Technical Design Report for the PANDA Internal Targets, (2012), https://fair-center.eu/fileadmin/fair/publications_exp/PANDA_Targets_TDR.pdf, (accessed 24 Feb 2020).

[46] J-PARC KOTO collaboration, T. Takahashi, ed., Write-ups for workshop on the project for the hadron experimental facility of J-PARC, Partial collection of LOIs at the extended hadron hall and the related topics, arXiv: 1906.02357.

[47] PANDA collaboration, Technical Design Report for the PANDA Solenoid and Dipole Spectrometer Magnets, arXiv: 0907.0169.

[48] PANDA collaboration, Superconducting solenoid for PANDA detector, International Conference on Instrumentation for Colliding Beam Physics, Novosibirsk, Russia, 24-28 February, 2020.

[49] PANDA collaboration, Technical Design Report for the: PANDA Micro Vertex Detector, arXiv: 1207.6581.

[50] D. Calvo, P. De Remigis, M. Fisichella, R. Wheadon, A. Zambanini, S. Mattiazzo et al., Study of SEU effects in circuits developed in 110 nm CMOS technology, PoS TWEPP2019 (2020) 126.

[51] P. Moreira et al., The GBT Project, TWEPP09 (2009) 342.

[52] F. Faccio et al., FEAST2: A radiation and magnetic field tolerant Point-of-Load buck DC/DC converter, IEEE Radiation Effects Data Workshop 2015 (2015), DOI.

[53] PANDA collaboration, Technical design report for the PANDA (AntiProton Annihilations at Darmstadt) Straw Tube Tracker, Eur. Phys. J. A 49 (2013) 25 [arXiv: 1205 . 5441].

[54] PANDA collaboration, Technical Design Report for the PANDA Forward Tracker, (2018), https://panda.gsi.de/system/files/user_uploads/admin/RE-TDR-2017-001.pdf, (accessed 24 Feb 2020).

[55] D. Przyborowski et al., Development of a dedicated front-end electronics for straw tube trackers in the bar PANDA experiment, 2016 JINST 11 P08009.

[56] M. Traxler, The TRB/DiRICH FEE+Readout System, in DIRC2017: Workshop on fast Cherenkov detectors, Castle Rauischholzhausen, Giessen, Germany 2017. 
[57] PANDA collaboration, Technical Design Report for the PANDA Luminosity Detector, (2018), https://panda.gsi.de/system/files/user_uploads/m.fritsch/RE-TDR-2015-001_1.pdf, (accessed 24 Feb 2020).

[58] PANDA collaboration, The luminosity detector at PANDA by HV-MAPS sensors, International Conference on Instrumentation for Colliding Beam Physics, Novosibirsk, Russia, 24-28 February, 2020.

[59] PANDA collaboration, Mechanics and Cooling for the PANDA Luminosity Detector, International Conference on Instrumentation for Colliding Beam Physics, Novosibirsk, Russia, 24-28 February, 2020.

[60] PANDA collaboration, Technical Design Report for the:PANDA Barrel Time-of-Flight, (2016), https://panda.gsi.de/system/files/user_uploads/ken.suzuki/RE-TDR-2016-003_0.pdf, (accessed 24 Feb 2020).

[61] B. Singh et al., Technical design report for the PANDA Barrel DIRC detector, J. Phys. G 46 (2019) 045001.

[62] PANDA collaboration, Technical Design Report for the PANDA Endcap Disc DIRC, arXiv: 1912.12638

[63] PANDA collaboration, The Barrel and Endcap Disc DIRC at PANDA, International Conference on Instrumentation for Colliding Beam Physics, Novosibirsk, Russia, 24-28 February, 2020.

[64] PANDA collaboration, Particle Identification Algorithms for the Panda Barrel DIRC, International Conference on Instrumentation for Colliding Beam Physics, Novosibirsk, Russia, 24-28 February, 2020.

[65] PANDA collaboration, Technical Design Report for: PANDA Forward Time of Flight detector (FToF wall), (2018), https://panda.gsi.de/system/files/user_uploads/admin/RE-TDR-2016-004.pdf, (accessed 24 Feb 2020).

[66] PANDA collaboration, Forward RICH detector for the PANDA experiment, International Conference on Instrumentation for Colliding Beam Physics, Novosibirsk, Russia, 24-28 February, 2020.

[67] PANDA collaboration, Technical Design Report for PANDA Electromagnetic Calorimeter (EMC), arXiv: 0810.1216.

[68] H. Flemming and P. Wieczorek, Low noise preamplifier ASIC for the PANDA experiment, 2011 JINST 6 C12055.

[69] PANDA collaboration, Technical Design Report for the Panda Forward Spectrometer Calorimeter, arXiv: 1704.02713.

[70] PANDA collaboration, Technical Design Report for the PANDA Muon System, (2012), https://fair-center.eu/fileadmin/fair/publications_exp/Muon_TDR.pdf, (accessed 24 Feb 2020).

[71] PANDA collaboration, FPGA-based algorithms for feature extraction in the PANDA shashlyk calorimeter, International Conference on Instrumentation for Colliding Beam Physics, Novosibirsk, Russia, 24-28 February, 2020.

[72] M. Traxler et al., Current and Future FPGA-TDC Developments at GSI, International Conference on Instrumentation for Colliding Beam Physics, Novosibirsk, Russia, 24-28 February, 2020.

[73] EPICS at Argone, Experimental Physics and Industrial Control System, https://epics.anl.gov/index.php, (accessed 24 Feb 2020). 
[74] Q. Hu, Z. Bai, J. Ritman, J.-S. Wang, H.-G. Xu and G.-M. Yu, Simulation of proton-proton elastic scattering for the KOALA recoil detector, Nucl. Instrum. Meth. A 898 (2018) 133.

[75] S. Wolff et al., Feasibility Studies for a FAIR Phase-0 Project at MAMI, in DPG Spring Meeting 2018 - Hadronic and Nuclear Physics, Bochum, Germany (2018).

[76] W. Li and J. Schwiening, GlueX DIRC at JLab, International Conference on Instrumentation for Colliding Beam Physics, Novosibirsk, Russia, 24-28 February, 2020. 